\title{
Modelo de rotación de personal y prácticas organizacionales
}

\author{
Yazmin HeRnÁNDEZ CHÁveZ \\ Universidad Politécnica de Tlaxcala. México. \\ Yazmin.hernandez@uptlax.edu.mx \\ Gerardo Hernández Chávez \\ Universidad Politécnica de Tlaxcala. México. \\ Gerardo.hernandez@uptlax.edu.mx \\ Angélica Mendieta Ramírez \\ Universidad Autónoma de Puebla. México. \\ angelicamendietaramirez@yahoo.com.mx
}

\begin{abstract}
Resumen
La industria del vestido es global, pues sus actividades de producción y comercialización se encuentran diseminadas en todos los países del planeta. Sin embargo, una forma de reducir los costos de producción ha sido trasladar el ensamble a los países con bajo costo de mano de obra como México, lo cual se ha traducido en precariedad y exclusión social en algunos casos, originando una alta rotación de personal en esta industria. Por su parte, la actividad de la confección en el Estado de Tlaxcala es una de las más importantes por el número de empleos que genera y el de empresas establecidas de manera formal, aunque existe también un porcentaje importante de la población en este Estado que se dedica de manera informal a la confección de prendas de vestir, bajo la modalidad de la maquila domiciliaria.
\end{abstract}

Palabras clave: Rotación de personal, industria del vestido, productividad, Estado de Tlaxcala.

\section{Turnover model and organizational practices}

\begin{abstract}
The apparel industry is global, as their production and marketing activities are scattered in every country on the planet. However, one way to reduce production costs has been to move the assembly to countries with low labor costs such as Mexico, which has resulted in instability and social exclusion in some cases, resulting in a high turnover in this industry. For its part, the activity of the preparation in the state of Tlaxcala is one of the most significant for the number of jobs created and established companies formally, although there is also a significant percentage of the population in this state that is informally dedicated to making clothing, in the form of the maquila home.
\end{abstract}

Keywords: Staff turnover, garment industry, productivity, State of Tlaxcala.

\section{Referencia normalizada:}

Hernández Chávez, Y.; Hernández Chávez, G. y Mendieta Ramírez, A. (2013) Modelo de rotación de personal y prácticas organizacionales. Historia y Comunicación Social. Vol. 18. N ${ }^{\circ}$ Especial Diciembre. Págs. 837-863.

Sumario: 1. Introducción. 2. La rotación de personal. 3. La globalización de la producción de prendas de vestir. 4. La rotación personal. 5. La rotación y la insatisfacción laboral. 6. Conclusión. 7. Bibliografía. 


\section{Introducción}

La industria del vestido enfrenta un florecimiento mundial, como consecuencia de las demandas de la sociedad como son: la moda, los cambios de estación y los nuevos materiales, que representan un papel central en los gustos y preferencias de los consumidores. Sin embargo, se caracteriza por ser una de las actividades industriales más competidas y dinámicas del mundo, puesto que las empresas líderes -ubicadas principalmente en países desarrollados- controlan el diseño, la comercialización y la marca. Estas organizaciones, con el fin de reducir sus costos de producción, promueven mediante la externalización de las operaciones de ensamble, la incorporación de países periféricos o en desarrollo como México, porque les suministran mano de obra barata de baja calificación, bajos salarios y escasa o nula sindicalización, lo que se traduce en precariedad y exclusión social, dando origen a una elevada rotación de personal. Por lo tanto, podemos destacar que los trabajadores constituyen un grupo vulnerable para poder ejercer sus derechos colectivos. La industria textil y del vestido continúa siendo una actividad tradicional, razón por la cual sus trabajadores son los menos escolarizados, perciben menos ingresos y laboran jornadas de trabajo por encima o por debajo de la jornada legal.

Se observa que la rotación del personal está influenciada por la desmotivación y la insatisfacción laboral, por lo que se puede afirmar que los motivos que implican la salida de los trabajadores son fundamentalmente laborales y en la mayoría de los casos pueden ser detectados mediante el estudio de la fluctuación potencial, por lo que si se toman las medidas necesarias se puede reducir la rotación real.

La rotación de personal es uno de los fenómenos laborales que impactan negativamente a la productividad de las naciones y en algunos sectores de la economía, representa la pérdida de recursos financieros y humanos que no son fácilmente recuperables porque implica renovar esfuerzos de ciclos de capacitación, curvas prolongadas de aprendizaje y en ese camino, el desperdicio de recursos técnicos e insumos. En la industria textil, la salida de trabajadores impacta de forma sustantiva en la eficiencia de los ciclos productivos y su salida al mercado nacional e internacional. De ahí que sea necesario estudiar las condiciones laborales, sociales, culturales y organizacionales que generan este fenómeno en una entidad de la República Mexicana como Tlaxcala que ha fundado buena parte de su desarrollo económico en la industria textil.

\section{La rotación de personal}

La rotación de personal en general ha sido un aspecto de gran interés para los estudiosos que han intentado analizar este problema bajo diversas perspectivas como Lucker y Alvaréz (1985), Lucker (1987), Rodríguez (1988), English William e Ibarreche (1989) y William (1989). El término de rotación de personal se utiliza regularmente para definir la fluctuación de personal entre una organización y su ambiente. 
Teniendo en cuenta que Arrioja (1993) afirma que hasta la mitad de los años setenta, la mayor parte de las investigaciones eran bivariadas, con énfasis en la relación entre la satisfacción del empleo y la rotación. Asimismo, en un artículo de Porter y Steers (1973) se incluye una revisión de investigaciones previas y la clasificación de factores relacionados con la rotación de personal. Porter menciona los factores organizacionales, del ambiente, del empleo y personales como los principales causantes de la rotación de personal. Por otra parte, English Williams e Ibarreche (1989) encuentran una fuerte relación entre las variables de personalidad y la permanencia en el empleo y señalan que los trabajadores mexicanos perciben las variables personales y del lugar de trabajo en forma muy diferente de cómo son percibidas por sus contrapartes americanos.

English Williams e Ibarreche mencionan que si bien las tasas de rotación son altas, las maquiladoras poco han hecho con relación a ello debido a que los trabajos son simples y hay abundante mano de obra. Además, Rodríguez (1988) señala también que la rotación no afecta la productividad de las empresas por la vasta mano de obra que busca empleo.

Carrillo y Santibáñez (2001) enfatizan la necesidad de que en el contexto del sector maquilador la rotación sea administrada, debido a la multiplicidad de factores que contribuyen a la decisión individual de dejar el empleo y a la magnitud del problema. Para ellos, el problema de rotación de personal es prácticamente imposible de solucionar sin que afecte la localización de las plantas y rebasa incluso las posibilidades de las políticas gerenciales. Por su consiguiente, Ahr \&Ahr (2000) mencionan que desde los escritos de March \& Simon's, los investigadores se han enfocado en ver cómo la disponibilidad de empleos y la insatisfacción laboral interactúan para dar como resultado el comportamiento de la rotación. Ahr \& Ahr mencionan, por ejemplo, que bajo ciertas circunstancias la disponibilidad de varias alternativas de empleo estimula la insatisfacción laboral del trabajador en su empleo actual al crear expectativas que no se cumplen en éste.

De igual forma, estos investigadores mencionan que algunos estudios contemporáneos sobre rotación documentan a los empleados que dejan sus empleos sin tener uno de reemplazo ya preparado. Estos descubrimientos sugieren que la insatisfacción laboral es más importante que la disponibilidad de empleo al determinar si un empleado dejará su trabajo. Ahr \& Ahr mencionan, asimismo, que los estudios de investigación proveen importantes hallazgos sobre las razones por las que los empleados se quedan porque el costo de dejar su empleo actual es más alto de lo que están dispuestos a pagar (compromiso de continuidad). Aquellos empleados que demuestran actitudes y hábitos de trabajo más deseables por los gerentes tienden a ser los que permanecen porque así lo desean (compromiso afectivo).

En lo relacionado con las prácticas organizacionales, representan una amenaza potencial a la eficiencia y efectividad organizacional en la industria maquiladora debido a la centralización del poder que caracteriza a este tipo de empresas. En la industria maquiladora, el grado de centralización es sumamente alto debido a que 
en su mayor parte son los directivos extranjeros quienes tienen a su cargo la toma de decisiones (Kacmar, 1999). Cuando una práctica organizacional afecta el proceso de toma de decisiones, las decisiones resultantes pueden de ser de forma adversa si una sola persona decide, a diferencia de haber utilizado una forma de decisión más objetiva y funcional. La centralización puede ser definida como el grado de extensión en que el poder para tomar decisiones es distribuido por medio de la organización. Cuando se toma una decisión en el núcleo del rango más alto, se dice que el grado de centralización es alto.

El Modelo de la Percepción de las prácticas organizacionales debe ser moderado por el entendimiento de éstas. El entendimiento se refiere al grado de extensión en el que alguien entiende cómo y por qué las cosas pasan de la forma en que suceden en la organización. Esto es, un individuo que tiene un entendimiento claro de quien es el responsable de la toma de decisiones y por qué fue seleccionado para ello, tendría un entendimiento más claro que alguien que no entiende ese proceso en la organización.

Esta influencia moderadora determina el grado de extensión en el cual las prácticas organizacionales son percibidas como una oportunidad o como una amenaza por los individuos. En general, cuando la percepción de éstas es alta y el entendimiento es bajo las prácticas organizacionales serán vistas como una amenaza, lo cual puede ocasionar reacciones negativas tales como ansiedad y mayor índice de rotación. Asimismo, cuando la percepción de éstas es alta y el entendimiento es alto también, las prácticas organizacionales serán percibidas como una oportunidad y las reacciones serán menos negativas. De igual manera, la forma en que un obrero percibe la aplicación de las prácticas organizacionales está influenciada por los factores personales y del entorno los cuales a su vez afectan la reacción del individuo hacia la organización, lo que causa retraimiento, ansiedad o compromiso y satisfacción. De acuerdo con Kracmar Michelle (1999) la percepción de las prácticas organizacionales es afectada por actividades tales como favoritismo, supresión de entidades de competencia y manipulación de políticas internas, según como el individuo las perciba.

En la industria maquiladora del norte de México muy a menudo el obrero no percibe las políticas organizacionales como justas y su aplicación como imparcial, por lo que el intercambio entre los supervisores y sus subalternos no se da en un ámbito de respeto en donde el individuo tenga la facultad de involucrarse en la toma de decisiones de la organización y percibir en ella en que las oportunidades y las formas de remuneración, así como las relaciones se dan en un ambiente de imparcialidad. Con respecto a la presente investigación se plantea la siguiente pregunta de investigación: ¿Cuál es la relación entre la rotación de personal y la percepción de los obreros de las prácticas organizacionales en la industria del vestido en el estado de Tlaxcala? 


\section{La globalización de la producción de prendas de vestir}

La industria del vestido es una de las más flexibles y con mayor capacidad de movilidad. Desde hace más de 30 años, la producción se traslada a otros países con el objetivo principal de disminuir costos. Esto ha sido posible gracias a la mejora de los transportes y las comunicaciones y, sobre todo, a la liberalización comercial. El cambio de las reglas en el comercio mundial significa que los países están aumentando su producción con el principal objetivo de exportar, y no para su propio consumo.

La pauta que se sigue a la hora de subcontratar la producción es la de buscar una localización más ventajosa y costos laborales más bajos. El sistema más corriente consiste en una fabricación triangular, en el que las fábricas del vestido, de los países con menor costo de mano de obra reciben encargos de Europa (EU) y Estados Unidos de América (EUA) principalmente, contratándoles su producción y embarcando posteriormente los productos ya confeccionados hacia los países compradores.

Las ventajas de subcontratar la producción a otros fabricantes son claras, ya que se puede hacer frente más fácilmente a los cambios del mercado, con una menor responsabilidad y manteniendo los costos al mínimo. Por lo que, las pautas de producción internacionales reflejan las estrategias cambiantes de las compañías en lo alto de la cadena productiva. Habitualmente, tienen sus sedes en EUA o EU, desde donde gestionan sus operaciones globales. La mayoría de estas empresas sólo venden, y sólo algunas también fabrican, aunque las diferencias entre ellas son cada vez más difusas. Entre los diferentes tipos de relaciones que se establecen entre los países capitalistas desarrollados y en desarrollo se presenta la subcontratación internacional, gracias a la cual algunas empresas importantes pueden reducir sus costos de producción y permanecer en el proceso productivo para competir en el mercado mundial (Alvarado Rosas y Vieyra, 2004; Caderot, 2004).

El aumento de la subcontratación está fuertemente ligado también a la necesidad de una rápida reacción a los cambios en las modas. La aplicación de nuevas tecnologías en el punto de venta ha permitido a los vendedores predecir tendencias y buscar una respuesta rápida de los fabricantes. El tradicional ciclo de dos temporadas, primavera-verano y otoño-invierno se ha dejado atrás, y el diseño, las telas y los colores se cambian con una frecuencia mucho mayor. Para los fabricantes, esto significa plazos de entrega más reducidos y la necesidad de una producción mucho más flexible.

Las demandas de la industria de la moda han actuado a veces en contra de la producción internacional y han permitido sobrevivir a algunos fabricantes de ropa en países con mayores recursos. Ahí donde los productos más básicos son fabricados fácilmente en países más pobres, los artículos de moda deben ser distribuidos más eficientemente en los países de venta. De nuevo, esto se consigue a través de un nuevo proceso de subcontratación que permite que los riesgos y las inseguridades estén bajo control.

Por lo que la subcontratación se ha convertido en una característica intrínseca de la industria de la confección, tanto en los países más desarrollados como en los menos. 
Sin embargo, no existe un patrón universal que explique cómo se opera. Las empresas subcontratadas pueden hacerse cargo de una línea entera de ropa o tan sólo de una tarea muy específica. Pueden utilizar sus propias materias primas o proveerse en la fábrica donde subcontratan y puede ser realizado en su hogar o en la fábrica y representa un vínculo entre el modo de producción formal y el informal. A su vez, las cadenas de subcontratación locales pueden proveer a un mercado doméstico o pueden estar ligadas a cadenas internacionales de oferta.

Estos cambios han significado una creciente presión sobre las firmas manufactureras, las cuales se ven obligadas a innovar sus propias estructuras industriales para desarrollar una mayor flexibilidad y capacidad de respuesta ante los requerimientos de calidad, velocidad y cambios continuos de estilo, así como a establecer alianzas estratégicas con sus clientes minoristas tendientes a lograr un mayor grado de eficiencia, coordinación y servicio dentro de la cadena de abastecimiento.

De ahí que la innovación vista desde la perspectiva territorial pueda ser considerada como un sistema dinámico de reproducción territorial fundado en innovaciones permanentes, resultado de relaciones de cooperación entre los actores, públicos o privados, individuales y colectivos de determinada región o territorio (Rodríguez, Pinto y Núñez, 2012: 67). Consiste en la capacidad de generar e incorporar conocimientos para dar respuestas creativas a los problemas del presente, no sólo en términos de crecimiento económico, sino desde una perspectiva integrada (Rambo y Filippi, 2009), a partir de E. Méndez, (2000) y Fernández (2004).

\subsection{El estado de Tlaxcala}

El estado de Tlaxcala se localiza en la meseta central del país, en una posición estratégica entre el Distrito Federal y los estados de México y Puebla, principales centros de consumo y el puerto de Veracruz, el más importante de México en materia de tránsito de mercancías, tanto de exportación como de importación. Tiene una superficie de 4,060.9 $\mathrm{km}^{2}$ y está integrado por 60 municipios con 1,180 localidades en total. Representa el $0.2 \%$ del territorio nacional y es la entidad más pequeña de la República Mexicana. 
Figura 1. El estado de Tlaxcala

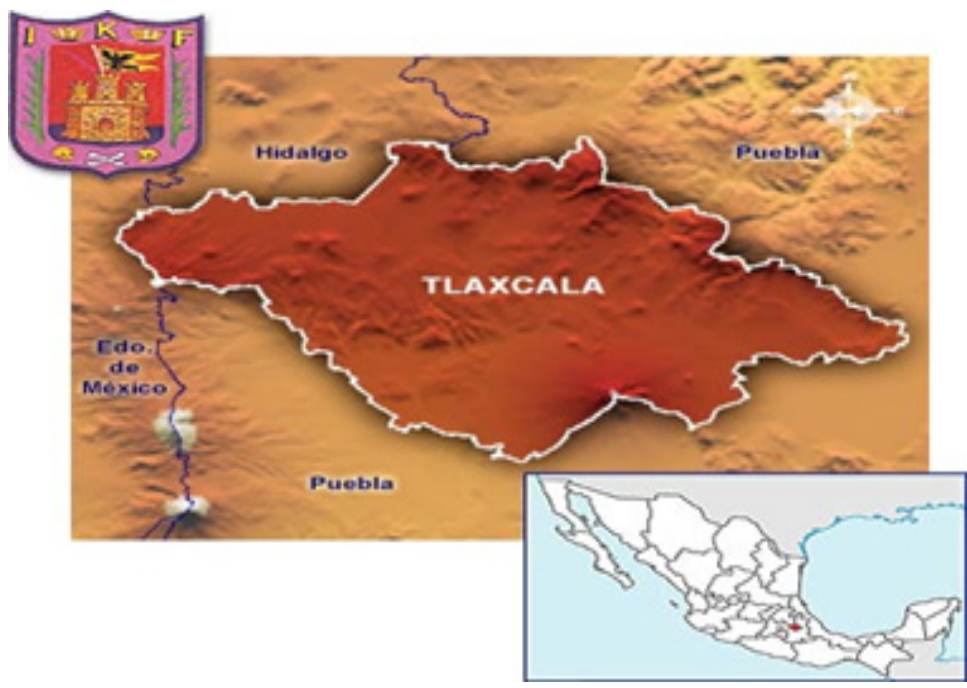

Fuente: INEGI (2013)

3.2 El sector de prendas de vestir en Tlaxcala y su contribución al PIB nacional

El Estado de Tlaxcala emplea 431395 el personal ocupado en la entidad, son hombres 264020 y mujeres 167375 (INEGI 2012). Es la segunda fuerza concentradora de empleos en la Cadena Textil-Vestido con 24\%. De acuerdo a las siguientes cifras, el perfil económico de Tlaxcala establece que cuenta con 1.2 millones de habitantes, que representan el $1.1 \%$ del total nacional, que lo ubica con la vigésima octava entidad en cuanto a población en 2012. (CONAPO, 2012) participando con el $1 \%$ del PIB Nacional y el $4.5 \%$ manufacturero (INEGI, 2012). Siendo el $26^{\circ}$ lugar para hacer negocios, cuenta con empresas en la transformación de 836 empresas que es el $0.7 \%$ del nacional, quedando en el $28^{\circ}$ lugar, así como los empleos en la transformación de 40.678 empleos que es el $0.9 \%$ del nacional y el $20^{\circ}$ lugar (IMSS, 2012), ocupa la posición $19^{\circ}$ de competitividad Estatal (IMCO, 2012) con una inversión extranjera directa de 14 millones de dólares de $0.10 \%$ del nacional ocupando el $23^{\circ}$ lugar, presentando la siguiente estructura (ver tabla 1) (Fuente: SE 2012).

Tabla 1. Estructura Productiva del Estado de Tlaxcala

\begin{tabular}{|l|l|}
\hline Sector & Participación \\
\hline Primario & $6.5 \%$ \\
\hline $\begin{array}{l}\text { Manufactura y } \\
\text { Transformación }\end{array}$ & $32.3 \%$ \\
\hline Sector Servicios & $61.1 \%$ \\
\hline
\end{tabular}

Fuente: (INEGI) sector manufacturero, 2012 
La Industria del Vestido en Tlaxcala dentro de la Cadena Textil-Vestido-Cuero, contribuye con el $2.1 \%$ del PIB sectorial nacional, ubicado como la $11^{\circ}$ entidad en cuanto a su contribución a este rubro, debajo de Guanajuato (19.7\%), Estado de México (14.1\%), Jalisco (7.3\%), Puebla (6.6\%), Hidalgo (6.6\%), Coahuila (4.1\%), Nuevo León (3.7\%), Aguascalientes (30\%), Yucatán (2.8\%). (Fuente INEGI 2012).

El Sector de la Industrial Textil y Vestido en el Estado de Tlaxcala, es un sector estratégico por su importante contribución a:

La producción ya que representa el $4.5 \%$ del PIB manufacturero y $1 \%$ del nacional.

Exportaciones: exporta aproximadamente $\$ 4,000 \mathrm{mdd}$, que representan el $2 \%$ de las exportaciones manufactureras. (AGA 2012)

Balance comercial: tiene cerca de $\$ 2000$ mdd de superávit. (AGA, 2012)

Es el Quinto proveedor de ropa a EUA: con una participación del 5\% en las importaciones totales de ropa de la Unión Americana (OTEXA, 2012)

Es el primer exportador latinoamericano de prendas de vestir a Norteamérica. (COMTRADE, 2012).

La venta de ropa, en el mercado interno, cercanas a los 57,000 mdp (ANTAD, 2012)

Es el décimo proveedor mundial de prendas de vestir con 13\% de las exportaciones mundiales de las exportaciones mundiales totales. (COMTRADE, 2012)

Es la séptima actividad generadora de valor: con un aporte de 56,000 millones de pesos al PIB Manufacturero (INEGI, 2012)

Es la Quinta fuerza concentradora de empleos de prendas de vestir: con más de 320 mil plazas laborales representando el 7.5\% del total manufacturero. (IMSS, 2012)

Es la segunda actividad generadora de empresas INMEX: con 486 empresas, representando el 7.3\% del total nacional. (Fuente: SE 2012)

\subsection{Aportación al empleo}

En la generación de empleo, aporta el 8\% del empleo manufacturero y el 2\% del empleo nacional. Participando con más de 306,425 mil plazas laborales directas y cercas de 1,000,000 indirectas. (IMSS 2011). Es el principal empleador de mano de obra femenina en el sector manufacturero, con cerca del $70 \%$ de contribución.

Con 8,980 empresas formales del Estado de Tlaxcala representa el $81 \%$ de la nacional (IMSS, marzo 2011). Siendo la segunda actividad generadora de empresas INMEX a nivel nacional, con el 8\%. (Fuente: SE, marzo 2011). En el comercio, las exportaciones de ropa de mayor peso en la cadena Fibra.Textil-Vestido: En 2000, la Industria del Vestido exportó $\$ 2,700$ mdd de productos confeccionados al mundo, por encima del Textil que exportó \$581 mdd y la fibra que exportó \$ 154.5 mdd. 
El empleo del sector vestido se concentra principalmente en las empresas grandes 4,628 (69\%), medianas $1,116(17 \%)$, pequeñas $683(80 \%)$, micro $306(5 \%)$ con un total de 6,733 empleos. (Fuente: IMSS, Mayo 2012). Mientras que en las empresas del sector vestido son predominante las Mipymes. En abril de 2011, las Micro, Pequeñas y Medianas Empresas MiPyME's del sector vestido que representan en promedio, el 93\% del total sectorial en Puebla - Tlaxcala reportaron el cierre de 33 empresas, lo que implica una caída, promedio del $3.5 \%$ de la planta fabril del sector en la región, como se muestra en la siguiente tabla:

Tabla 2. Estructura MiPyME's del Sector Vestido en Edo. De Tlaxcala

\begin{tabular}{|c|c|c|c|c|}
\hline Estrato & $\begin{array}{c}\text { Empresas (Abril } \\
\text { 2011) }\end{array}$ & Participación (\%) & Número & Porcentual \\
\hline Micro & $80 \%$ & $63 \%$ & 0 & $0 \%$ \\
\hline Pequeña & $27 \%$ & $21 \%$ & -4 & $-13 \%$ \\
\hline Mediana & 13 & $10 \%$ & $-1 \%$ & $-7 \%$ \\
\hline Grande & 7 & $6 \%$ & 1 & $17 \%$ \\
\hline TOTAL & $\mathbf{1 2 7}$ & $\mathbf{1 0 0 \%}$ & $\mathbf{- 4 \%}$ & $\mathbf{- 3 \%}$ \\
\hline
\end{tabular}

Fuente: IMSS, 2011

Algunas de las características más destacadas de la industria del vestido en el estado son:

La tecnología utilizada por las grandes empresas en la confección es, en términos generales, maquinaria moderna; las pequeñas y medianas empresas cuentan con maquinaria no actual;

La capacitación de los obreros se da principalmente a través del aprendizaje en el trabajo, donde, incluso los técnicos de mantenimiento carecen de entrenamiento específico;

La escasez de mano de obra es un efecto por la atracción que ejercen las grandes empresas maquiladoras por los salarios que ofrecen; sin embargo, las condiciones de empleo son precarias;

Las funciones técnicas y administrativas, están delimitadas en las grandes empresas y se tiene personal ocupado en cada una de esas áreas. En las pequeñas empresas, el dueño realiza la administración;

La asesoría técnica es limitada y llega a pocas empresas. Las grandes y medianas empresas reciben únicamente si forman parte de alguna asociación o, en el caso de las maquiladoras, por depender de empresas externas.

Las posibilidades de recibir en micro y pequeñas empresas son bastante distantes, ya que en ocasiones adquieren maquinaria usada; 
En las pequeñas empresas la mano de obra representa el 6\% del costo y en las grandes sube a $80 \%$, debido a los mayores sueldos y salarios que pagan.

Con respecto a los trabajadores existe una alta rotación de personal por las condiciones mínimas de trabajo, además de discriminación y maltrato hacia las mujeres (quienes son el $80 \%$ de mano de obra), así como explotación y salarios bajos. Como se observa todo esto hace que la industria de la confección esté en crisis.

A pesar de la gran solidez que ha presentado la región Puebla -Tlaxcala, la industria del vestido enfrenta múltiples factores que han limitado de manera importante su desarrollo y competitividad. Se estima que de 6 de cada 10 prendas que se consumen en nuestro mercado son de procedencia ilegal (piratería, contrabando, robada, etc) lo que ocasiona: pérdida de miles de empleos, el cierre de empresas formales, proliferación de la clandestinidad, pérdidas millonarias al erario y descomposición del tejido social.

De acuerdo a la American Chamber, en 2011 las pérdidas proyectadas por concepto de piratería en todos los productos ascenderán a $\$ 983,182$ millones de pesos (mdp) en donde los productos más sensibles a este ilícito son los cds, dv's, ropa, calzado, accesorios, software y los juguetes.

En la industria del vestido el fenómeno de la piratería ha repercutido de manera drástica en el desarrollo de este sector, de acuerdo a cifras de la CANAIVE 2012, las prendas de vestir fueron el producto que más resintió el fenómeno de la piratería en términos de valor, la ropa el $8 \%$ de participación porcentual.

Por lo podemos decir, que la industria del vestido es una de las más flexibles y con una alta movilidad, ya que la producción se traslada con facilidad a otros países con el objetivo de disminuir costos de producción por ser intensiva en mano de obra, a fin de mejorar su competitividad en el mercado mundial. En el caso de Tlaxcala ésta industria es la segunda fuerza concentradora de empleos, en cuanto al PIB manufacturero aporta el 4.5 por ciento y el 1 por ciento nacional de ahí resalta su importancia de atender su problemática, que en caso particular propone un modelo teórico para entender la rotación de personal y su relación con las prácticas organizacionales, que a continuación se abordaran.

\section{La Rotación de Personal}

La Rotación de Personal es un factor que tiene gran impacto, para los empresarios, ya que cualquier estrategia de calidad, productividad o mejoría en el empleo requiere de mano de obra con cierta estabilidad, para la Secretaría de Trabajo y Previsión Social, porque las políticas de empleo, sobre todo de capacitación, pueden tener un barril sin fondo ante una mano de obra con gran movilidad; para la sociedad en su conjunto, debido a que las habilidades, los conocimientos y las destrezas adquiridos en este tipo de empleo son perdidos por el desplazamiento de los trabajadores 
hacia otros sectores productivos o fuera de la actividad económica y para los propios trabajadores, finalmente, puesto que no desarrollan en forma plena la solidaridad de grupo ni capitalizan el conocimiento adquirido. (Hernández, Y. Hernández G y Rojas J. 2008)

La Fidelidad de la organización (Chiavenato I, 2009) contribuye a reducir la rotación de personal (turnover), es decir, el flujo constante de salidas (renuncias o despidos) y entradas (admisiones) de personas a la organización, o el ingreso de personas para compensar las salidas de integrantes de la organización. La rotación (Tamayo P, 2008) puede ser voluntaria (cuando la persona decide separarse de la empresa) o involuntaria (cuando la empresa decide despedir a la persona, sea por reducción de personal o por desempeño insuficiente). El aumento de la rotación implica un incremento de los costos de admisión, como reclutamiento, selección y capacitación, así como los costos por separación, como indeminización, anticipo de días festivos y pago de vacaciones. ¿De cuánto son dichos costos? Son mayores de los que se piensa ( Mercer M. 1988, Cascio W.F, 1991, Chiavenato 2004, Gómez Mejía L, Balkin D y Cardy R. 2008). Por ejemplo, el costo en que incurre una compañía común de tecnología de la información en Estados Unidos por reemplazar a un programador o analista de sistemas se estima en $\$ 34,100$; y se ha calculado que el de una tienda al menudeo por las ventas perdidas al sustituir a un empleado es de $\$ 10,445$. Así como, el costo promedio de rotación por persona es de alrededor de $\$ 15,000$ dólares. Cuando un persona sale de la organización es necesario sustituirla, cosa que no siempre es fácil ni inmediata. Por tal razón, la rotación perjudica la eficiencia de la organización y puede indicar que se está perdiendo capital humano que se va a otras organizaciones. Por otra parte, un índice de rotación de cero (rotación nula) puede tener un significado negativo, es decir, la organización no renueva a sus participantes y se vuelve rígida e inmutable. Debe existir cierta rotación a efecto de sustituir a personas que tienen un desempeño inferior por otras más competentes y motivadas y así renovar y traer ideas nuevas a la organización. Las organizaciones que están más expuestas a los cambios del entorno (como las industrias de la electrónica, la moda, los medios y la publicidad) necesitan renovarse constantemente y requieren una mayor movilidad de personal. En el mundo laboral cambiante de hoy, los niveles de rotación iniciada por los empleados facilitan la flexibilidad organizacional y la independencia de éstos, disminuye también la necesidad de despidos de personal llevados a cabo por la administración. Una cierta rotación voluntaria de los trabajadores permite aumentar la flexibilidad de la organización o reducir la necesidad de despidos en periodos de baja actividad.

Sin embargo, es frecuente que la rotación involucre la pérdida de personas que la organización no desea perder. Por ejemplo, un estudio de 900 empleados que habían renunciado a sus trabajos descubrió que el $92 \%$ obtenía de sus superiores calificaciones de "satisfactorio" y aún mejores por su desempeño. (citado en "You Often Lose the Ones You Love" Industry Week, 21 de noviembre de 1988) Por tanto, cuando la rotación es excesiva o involucra trabajadores valiosos, es un factor de ruptura que dificulta la eficacia organizacional. 
En general, cuando las organizaciones tienen que recortar costos o reducir su tamaño para adaptarse a la contracción del mercado, ofrecen programas de renuncia voluntaria. Aunque, hay una concepción generalizada de que los trabajadores abandonan su empleo por una situación problemática, se parte de que el abandono obedece a que algo no les gusta, les parece mal o porque no tiene sentido quedarse en el empleo. (Robbins P. S. y Judge A.T, 2009)

En este marco, algunas de las ideas más extendidas sobre la rotación de los trabajadores son las siguientes, porque: los salarios son bajos y las prestaciones bajas; su trabajo es aburrido y cansado; los trabajadores no son tomados en cuentan en las decisiones de la empresa; no hay un ambiente agradable en el empleo; hay una excesiva demanda de empleo y una escasa mano de obra disponible.

Independientemente de sus determinantes, esta movilidad de la mano de obra es uno de los problemas más serios que aquejan actualmente a la industria del vestido, porque les suministran mano de obra barata de baja calificación, bajos salarios y escasa o nula sindicalización, con las consecuentes precariedad y exclusión social, da origen una elevada rotación de personal. La rotación de personal está influida por la desmotivación y la insatisfacción laboral, por lo que los motivos que implican la salida de los trabajadores son fundamentalmente laborales y en la mayoría de los casos pueden ser detectados mediante el estudio de la fluctuación potencial, así que, si se toman las medidas necesarias, se puede reducir la rotación de personal. El fenómeno de la rotación de personal para los empresarios es un problema, ya que cualquier estrategia de calidad, productividad o mejoría en el empleo requiere de mano de obra de cierta estabilidad.

\subsection{Diferentes teorías y modelos sobre la rotación de personal}

La investigación realizada sobre la rotación no es muy abundante y se ha centrado principalmente en tratar de identificar las variables relevantes que influyen en ella, para tratar de controlarla y reducirla. En la revisión de la literatura que trata sobre la relación existente entre rotación y satisfacción laboral, se constata una significativa asociación negativa entre ambas variables (Mobley, 1977). Por tanto, junto a la satisfacción laboral, se considera la influencia que ejercen otras agrupaciones de variables sobre la rotación, como pueden ser las variables organizativas o las variables de carácter económico y ocupacional.

\subsection{Modelos de decisión de rotación}

En primer lugar, March y Simon (1958) sugerían que la marcha voluntaria del individuo de la organización, dependerá de la percepción individual que este tenga sobre sus propios deseos de realizar el cambio y de la facilidad con que pueda ejecutar dicho cambio. En el establecimiento del criterio de rotación adoptado, se incluyen los componentes intraorganizacionales que les sean accesibles.

Además, este modelo ha sido considerado como uno de los primeros trabajos formales en el estudio de rotación de personal, por ser el primero en abordar el 
problema con un enfoque integral. El haber tomado en cuenta la satisfacción laboral y el entorno económico como factores claves dando las bases para teorías posteriores con enfoque sistémico. Este modelo también se orienta en observar el proceso de la rotación de personal a partir de la decisión propia del trabajador de salirse de una empresa, por lo que la rotación de personal en este modelo es entendida como "el retiro voluntario de un individuo de abandonar una organización de la cual recibe una compensación económica". Su modelo se compone de dos conceptos primordiales:

La intención del trabajador de salirse de la organización.

La percepción de oportunidades laborales externas.

La satisfacción laboral es definida como "el estado de percepción que tiene el trabajador de la organización del trato que recibe de sus compañeros de trabajo, y de su participación, en su actividad laboral”. La satisfacción laboral está en función de las variables: conformidad con el trabajo, relaciones laborales y la identificación con el trabajo, las que se encuentran presentes en toda clase y tamaño de organización. En suma el modelo de March (19589 ha otorgado una base sólida para muchos de los trabajos posteriores sobre la rotación de personal, por el enfoque de equilibrio en la evaluación integral del mercado laboral y de la satisfacción. Por su parte Vroom (1964) trata de dar una explicación plausible al modo en que el individuo decide abandonar la empresa, desde la teoría de la Instrumentalidad-Valencia.

\section{a) Porter y Steers (1973)}

Apunta hacia la centralidad que las expectativas de cada individuo tienen sobre la decisión de abandono. Es decir cada sujeto enfoca su propia situación de empleo bajo una serie de expectativas, referidas a su trabajo actual, al sueldo que percibe, a la promoción a la que tiene acceso, a la supervisión que recibe de sus superiores y a las relaciones interpersonales (ricas o pobres) que mantiene con su grupo de trabajo.

\section{b) Modelo de Mobley (1977)}

El modelo de Mobley (1977) tiene influencia de las investigaciones de March y Simon (1958) y Prince (1977). Mobley (1977) se preocupó por encontrar la explicación de cómo el trabajador percibe y evalúa a la satisfacción al igual que al medio ambiente laboral y como los aspectos individuales afectan a dicha percepción. Mobley (1977) define la rotación de s como "el retiro voluntario e involuntario de una persona de una organización de la cual recibe un salario". Dentro de la Definición no se consideran los movimientos internos o transferencias de una organización y se hace énfasis en el retiro voluntario o involuntario, la separación voluntaria se inicia por parte del empleado, y el retiro involuntario lo emprende la organización. Las consecuencias negativas de la rotación de personal, al amparo de este modelo son: los costos, perturbación en el desempeño, perturbación en los aspectos sociales y de comunicación, baja moral e indiferencia en las estrategias de control. Y las consecuencias positivas son: desplazamiento de trabajadores con bajo desempeño, innovación, flexibilidad y adaptabilidad y reducción de conflictos en el ambiente laboral. Sostiene que los costos del cambio pueden ser altos o bajos. Si son altos el 
individuo revaluará de nuevo su trabajo actual, limitando su forma de pensar sobre la convivencia de su marcha o adoptando otras estrategias de conducta de abandono (retrasos, absentismo, etc) si la percepción de cambio encuentra alternativas de costos no prohibitivos, investigará exhaustivamente la alternativa concreta, evaluando-comparando el trabajo actual con la alternativa estudiada. Si finalmente, no la encuentra aceptable, reevaluará su posición actual, continuando la búsqueda o se desviará hacia otras formas indirectas de abandono. Si la comparación resulta favorable a la nueva alternativa considerada, se estimulará la intención de marcha.

Este modelo se reconoce que existen diferentes individuales en la secuencia de las fases que sigue cada sujeto para llegar a la decisión de rotación. Es más un modelo heurístico que descriptivo, en el que predominan los componentes de tipo impulsivo que los de corte racional.

\section{c) Modelo de Price (1977)}

Este Modelo toma de March (1958) las variables del salario y las relaciones laborales para incluirlas como parte de sus elementos generadores de la satisfacción a la que define como el "grado de actitud positiva que tiene el trabajador hacia la organización" . las variables de acuerdo al modelo de Price (1977) que conciben a la satisfacción son:

El salario (el pago económico que recibe el trabajador por su labor).

La integración (la participación del trabajador en las decisiones sobre su tarea y en los objetivos de la organización).

La comunicación informal (la que recibe el trabajador sobre su desempeño a través de sus compañeros).

Comunicación formal (la que recibe el trabajador oficialmente por parte de la organización en relación con su desempeño), y

La centralización (el nivel en el cual se toman las decisiones que afectan la operatividad de la organización).

La aportación de Price (1977) al estudio de la rotación de personal es su contribución positiva al integrar las variables organizacionales del salario, integración, comunicación informal, comunicación formal y centralización como determinantes de la satisfacción y está a su vez, de la decisión del trabajador de permanecer o retirarse de la organización. La decisión, por parte del trabajador, de retirarse de la empresa puede verse afectada de forma positiva o negativa dependiendo las opciones que el trabajador encuentre en el mercado laboral. El modelo asume que la satisfacción es evaluada por los empleados conforme a sus percepciones hacia la organización y que los individuos tienen conocimiento de las alternativas que ofrece el medio ambiente y nos son restringidos a obtenerlas (Griffeth y Hom, 1995). Al modelo de Prince (1977) se le realizaron cinco pruebas científicas por Bluedorn (1980), y en todas las pruebas no se encontró una interacción entre la satisfacción y las oportunidades, por lo que Bluedorn (1980) concluyó que las oportunidades actúan como pronosticador 
de la satisfacción y no como una variable de intervención entre la satisfacción y la rotación de personal.

\section{d) Modelo de inversión de Farrell y Rusbult (1981)}

Farell y Rusbult (1981) derivaron su modelo de cambios sociales de Homans (1961) y de la teoría de Thibaut y Kelley (1959) y Kelley y Thibaut (1978) e intentaron explicar el Compromiso Organizacional, entendiendo por ello: "El estado de obligación del individuo hacia el resultado de su comportamiento" y este compromiso fue relacionado a la probabilidad de que un empleado deje su trabajo o influya en él a sentir sentimientos de unión y afecto hacia la organización. Por lo tanto, el grado de compromiso puede reflejar la intención de permanecer o abandonar a una organización.

El modelo de Farrel y Rusbult (1981) fue probado por ellos mismos en una simulación de trabajo dentro de un laboratorio y con una investigación local en trabajadores del sector industrial. Los resultados mostraron quela recompensa laboral y los costos del trabajo predicen fuertemente la satisfacción laboral. Asimismo la combinación de recompensas, el valor de los costos, el valor de las alternativas y el tamaño de la inversión predicen fuertemente el compromiso del trabajo y éste, a su vez predice la rotación de personal.

\section{e) Modelo de la Multi-ruta de Steers y Mowday (1981)}

El análisis que realizan Steers y Mowday (1981) se estructura en tres segmentos: origen de la expectativas y actitudes del trabajo; cómo las actitudes afectan la intención de abandonar un trabajo y el proceso por el cual la intención de abandonar un trabajo conduce a la rotación de personal. En suma, el modelo de Steers y Mowday (1981) es una compleja representación del proceso de la rotación de personal que marcó muchos constructor innovadores, incluyendo la noción largamente olvidada de que los esfuerzos para cambiar el medio ambiente laboral podría interrumpir el proceso por el cuál la insatisfacción laboral promueve el retiro del trabajador. Los esfuerzos para cambiar el trabajo el trabajo también puede directamente afectar otras determinantes de la rotación de personal.

\section{f) Modelo de la cúspide de la catástrofe de Sheridan y Abelson (1983)}

Desviándose del pensamiento convencional, Sheridan y Abelson, desarrollaron el modelo que se basa en dos determinantes el compromiso organizacional y la tensión laboral que puede conducir a la conducta de abandonar el trabajo. Éste tiene tres características la primera es la conducta de dejar el trabajo considerada como una variable discontinua con cambios repentinos observados entre los diferentes estados de dicha conducta, donde presumiblemente el empleado intenta mantener su actual trabajo el mayor tiempo posible sin embargo, una vez acumulada la insatisfacción, como resultado de la disminución en el compromiso hacia la empresa o por el estrés laboral, el empleado repentinamente cambia del estado de permanecer a abandonarla. La segunda el modelo presenta una zona estéril de conducta que lo ocasiona el deseo de cambio y la tercera la conducta divergente que ocurre cuando llega a la cúspide 
el nivel de estrés y tensión laboral. En suma el modelo de la cúspide de la catástrofe es considerado como uno de los principales adelantos en el estudio de la rotación de personal, presentando al retiro por parte del empleado de una organización como una variable discontinua que podría predecir ls terminación laboral. Bajo este modelo la resignación es observada como una manifestación de evitar el trabajo y la rotación de personal es considerada una evolución de conducta menos extrema, en relación al ausentismo y al pobre desempeño.

El modelo de la cúspide de la catástrofe amerita, sin embargo, más trabajo empírico y teórico debido a que sus dos determinantes (tensión laboral y compromiso) capturan de manera insuficiente las varias razones del porqué los empleados abandonan su trabajo.

\section{g) Modelo económico del trabajo de Hulin, Roznowski y Hachiya (1985)}

Revisando las pruebas empíricas de las alternativas del trabajo, Hulin, Roznowski y Hachiya (1985) concluyeron que la valoración de la percepción de la perspectiva del mercado laboral ha predecido pobremente la rotación de personal, mientras que las estadísticas del mercado laboral, tales como el índice de desempleo, pronostican con mejor consistencia la rotación de personal. Consideraron que las alternativas de trabjao puede directamente afectar la satisfacción laboral a diferencia del argumento de que la satisfacción laboral es la que influye en la búsqueda de alternativas laborales. También sostuvieron en su modelo que las oportunidades laborales pueden directamente inducir en la rotación de personal, debido a que los empleados dejan un trabajo cuando están seguros de una alternativa laboral y no por conjentura de que existe una probabilidad de trabajo. Toman en cuentan tres argumentos: las diferencias económicas producen diferentes fuerzas laborales. Las oportunidades laborales directamente influyen en la satisfacción laboral y estas afectan a la rotación de personal.

En suma, Hulin, Roznowski y Hachiya (1985) ofrecen una perspectiva de reexaminación del papel de las alternativas laborales en el proceso de retiro, resolviendo una vieja controversia en el estudio de la rotación de personal. Dan una invaluable taxonomía de las diferentes formas de abandonar un trabajo (retirarse de la organización o adoptar otro tipo de conductas que disminuyen el desempeño laboral) e incluyen en su modelo a los trabajadores marginales que siguen un camino distinto de la ruta convencional de insatisfacción laboral, búsqueda de trabajo, decisión de abandonar el trabajo y retiro (Mobley, 1977). Está teoría explica por que la insatisfacción en el trabajo invariablemente conduce al retiro por parte del trabajador de la organización, los empleados pueden responder a la insatisfacción no necesariamente retirándose de la empresa, pero sí tomando actitudes de disminuir su esfuerzo, sus habilidades, su tiempo dedicado a la organización (contribuciones laborales) o cambiando el actual trabajo por transferencias internas o buscar pertenecer y ocupar un puesto en algún sindicato. Para ellos, presumiblemente el retiro psicológico o el cambio de trabajo llegará a ser sustituido por el retiro del trabajador, a lo que vendría siendo la rotación de personal, como una entre muchas reacciones conductuales de la insatisfacción (Hulin, 1991). 
Una de las principales críticas al modelo de Hulin, Roznowky y Hachiya (1985) es que excluyen de su modelo algunos constructos importantes para entender la rotación de personal, como el compromiso hacia la organización y las influencias externas (Blau, 1993; Hom y Griffeth, 1991; Lee y Mowday, 1987 y Price y Mueller, 1986)

\section{h) Modelo de la motivación dinámica de Fichman (1988)}

La motivación dinámica de Fichman (1988) consiste en considerar que toda persona está motivada para realizar alguna acción y que la motivación puede variar en fuerza dependiendo de qué tan atractivo sean los resultados a obtener. Su punto más importante es la comprensión de las necesidades de los empleados para reducir la rotación de personal, por lo que utiliza la teoría de Maslow y la teoría económica para entender el fenómeno de la rotación de personal.

De la teoría de Maslow (1954) toma el concepto de que es importante identificar las necesidades del trabajador, las cuáles pueden clasificarse en:

Necesidades físicas básicas, llamadas necesidades primarias.

Necesidades sociales y psicológicas, llamadas necesidades secundarias.

Para Fichman (1988) las necesidades secundarias son las que complican los esfuerzos motivacionales de los administradores para manejar la rotación de personal, además de considerar las necesidades primarias como básicas para la retención de los trabajadores eficientes. De la teoría económica obtiene el concepto de costo de oportunidad, definiéndolo "como los costos de utilizar recursos de capital humano capacitados y experimentados para que rindan en un desempeño adecuado, que beneficie tanto a la organización como a la persona". Utilizar personal no adecuado en la organización representa un costo de eficiencia y eficacia, en la persona un costo de oportunidad al no haber ingresado en una organización conforme a sus conocimientos y aptitudes, al no haber tomado en cuenta lo anterior genera para la organización gastos innncecesarios en la contratación de nuevo personal y en la persona desmotivación y abandono de su trabajo. Estas aportaciones de Fichman (1988) ha generado investigaciones posteriores sobre los impulsos y las necesidades de los empleados con el objetivo de entender el complejo manejo de la rotación de personal.

Entre las investigaciones se encuentran las de McClelland (1961), quién elaboró, un esquema de clasificación en el que sobresalen tres de los impulsos más dominantes y en el que se señalaba la significación de éstos, para la motivación y su efecto en la rotación de personal. Las investigaciones de McClelland se centraron en los impulsos de logros, asociación y poder.

\section{i) Modelo del desdobles de Lee y Mitchell (1994)}

Lee y Mitchell (1994) considerando la teoría de imagen de Beach, (1990) como una manera de entender el proceso de la toma de decisión y de terminación. La teoría de imagen desafía el predominio de las teorías de rotación d epersonal que asumen que la toma de decisión de retirarse del trabajo tiene una base racional económica (Hulin, Roznowki y Hachiya, 1985; Mobley, Griffeth, Hand y Menglino, 1979) y 
presuponen que las personas toman decisiones al comparar el ajuste de las opciones en la decisión de varias imágenes interna, mas que en maximizar la expectativa subjetiva de la utilidad. La teoría de la imagen postula que las personas deben filtrar el constantemente bombardeo de información para seleccionar las opciones adecuadas. Esta proyección es rápida que requiere poco esfuerzo cognitivo y compara las características de las opciones de una a tres imágenes internas: valor (grupo de valores generales y estándares que lo definen a uno mismo); trayectoria (grupo de metas que energetizan y dirigen la conducta individual) y las estrategias (grupo de conductas tácticas y estrategias para unir las metas personales). Estas prueba de compatibilidad es no compensatoria y requiere que las opciones se ajusten en una o más imágenes. Si una opción conductual encuentra la prueba, la persona compara la alternativa con su statu quo. Usualmente las personas continúan con el statu quo, algunas veces llegan a seleccionar una conducta diferente. Si las numerosas opciones sobreviven en la proyección, una persona corre la prueba "carácter lucrativo" seleccionando la mejor alternativa de acuerdo al análisis de costo-beneficio.

Utilizando la teoría de imagen Lee y Mitchell (1994) proponen que el proceso completo de la proyección y la toma de decisiones comienzan con un "snock en el sistema", un evento específico que estremece al empleado para tomar juicios deliberados en relación con su trabajo y probablemente a considerar el retiro del trabajo. También establecieron que el contexto social y cognitivo que rodean experiencias del snock proveen un marco de decisiones o marco de referencias con el cuál se interpreta el snock en toda su dimensión, como una novedad, favoritismo ó amenaza.

\section{j) Modelo de medida estructural utilizando una muestra nacional de trabajadores (Lambert, Hogan y Barton, 2001)}

El modelo de Lambert, Hogan y Barton (2001) incorpora cuatro variables siendo las características demográficas el medio ambiente laboral, la satisfacción y la intención de rotación de personal, su modelo fue desarrollado y probado usando una muestra nacional de trabajadores estadunidenses, teniendo como resultado que el medio ambiente laboral es más importante en la formación de la satisfacción laboral para el trabajador que las características demográficas. Y que la satisfacción laboral es un antecedente muy importante en la intención de la rotación de personal, así mismo que la satisfacción laboral es la variable clave entre el medio ambiente laboral y la intención de rotación de personal.

El modelo tiene como base principalmente los dos factores antes señalados que influyen sobre la satisfacción laboral y la intención de rotación de personal, por lo que ellos los destacan como los cuatro factores de su estudio:

Las características demográficas

La satisfacción laboral

El medio ambiente laboral

La intención de rotación 
En suma consideraron que las características demográficas están comúnmente incluidas en los estudios sobre la satisfacción laboral como variables de control, de ahí la justificación en la inclusión dentro de su estudio de las variables de la edad, el género, la educación y la permanencia del empleado.

k) La Rotación de personal en los trabajadores de la información tecnológica: examinando empiricamente la influencia de las actitudes, las características del trabajo y los mercados externos (Thatcher, Stepina y Boyle 2002)

Thatcher, Stepina y Boyle (2002), elaboran un modelo conceptual que une las percepciones del medio ambiente en el trabajo interno y los mercados externos para los trabajadores de la información tecnológica, su modelo se enfoca en el compromiso organizacional como principal predictor de la intención de la rotación de personal, considerando que el compromiso organizacional media las percepciones del lugar de trabajo y el medio ambiente externo sobre la intención de rotación de personal.

En suma los resultados de Thatcher, Stepina y Boyle (2002) sugieren que la intención de la rotación de personal predice la rotación de personal y el compromiso organizacional tiene una relación negativa con la intención de rotación de personal, por otro lado la satisfacción laboral y la significancia en la tarea tuvieron un efecto positivo sobre el compromiso organizacional. También la significancia de la tarea, la variedad en la tarea y la autonomía tuvieron una influencia positiva en la satisfacción laboral. Aunque la percepción competitiva del salario demostró una relación débil con la satisfacción laboral, asimismo indicaron que las percepciones del mercado laboral tuvo una fuerte influencia positiva sobre la intención de rotación de personal.

\section{l) Modelo de sistemas (2004)}

El modelo de Mobley, Griffeth, Hand y Menglino (19799, ha servido como base para estudios posteriores sobre la rotación de personal, desde una perspectiva como sistema (Davis y Werther, 2004). El enfoque de sistemas parte de conceptualizar a la organización como un grupo de diversas actividades o entidades que están relacionadas entre sí, pero que poseen limites claros y precisos. Por lo tanto, para el enfoque de sistemas, una organización está compuesta de los sistemas de producción, de ventas, financiero-legal y administrativo, como se esquematiza en la Fig.2. Este último sistema se integra a su vez por dos subsistemas: el de recursos humanos y el de servicios, que comprende el resto de las actividades que no están directamente relacionadas con el manejo de los recursos humanos. El sistema de recursos humanos interactúa tanto con los sistemas internos de la organización como los externos, denominado ambiente. 
Fig. 1. Sistema de administraciones de operaciones.

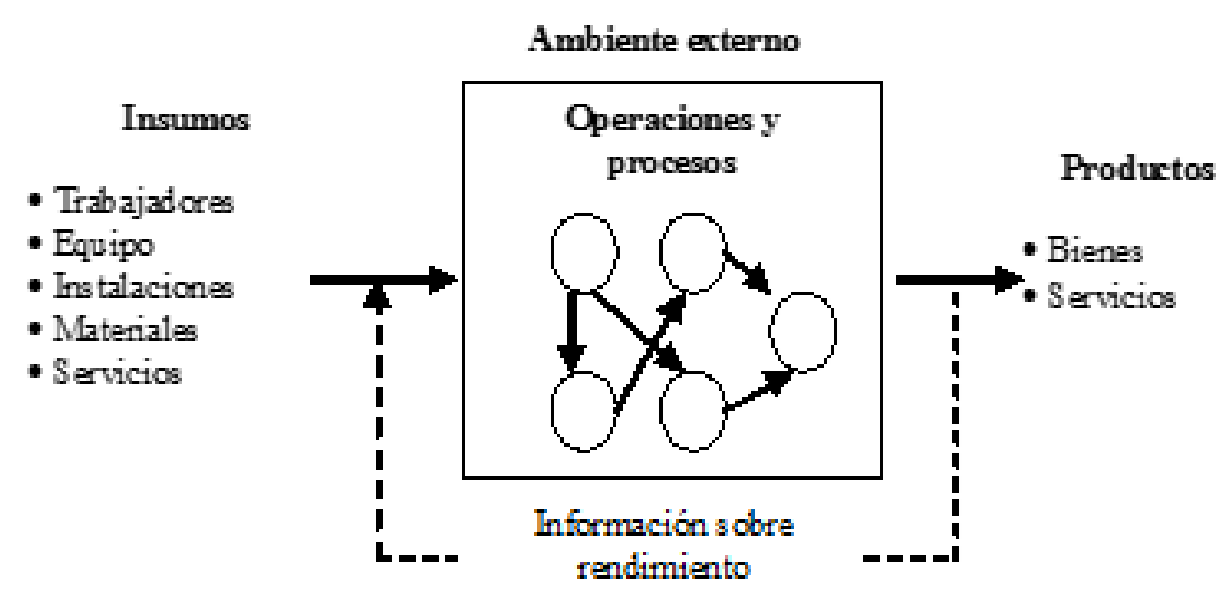

En este marco de sistemas, la actividad de la administración se centra en términos de requerir insumos, transformarlos y convertirlos en productos. El administrador verifica que sus acciones fueron adecuadas cuando los productos de su actividad son apropiados. Este proceso genera la retroalimentación, que es la posibilidad de contar con información para determinar el grado en que se tuvo un éxito o un fracaso. En el modelo de sistemas, el ambiente o entorno constituye un elemento importante, porque la mayor parte de los sistemas son abiertos (Tamayo, P. 2008). Un sistema abierto es el que puede ser afectado por el ambiente en el que opera. En una organización, los factores que inciden son: diversidad de la fuerza de trabajo; factores demográficos; aspectos económicos; aspectos culturales; aspectos tecnológicos y aspectos gubernamentales.

Entre el mercado de recursos humanos y el mercado de trabajo hay un continuo intercambio, donde interactúan recíprocamente y tienen una continua y mutua influencia, la oferta de una mercado corresponde a la demanda en el otro y viceversa; la salida de uno es la entrada del otro, lo que implica para la organización que los retiros de personal deben ser compensados con nuevas admisiones, a fin de mantener el nivel de recursos humanos en proporciones adecuadas para que opere el sistema. Para el sistema la relación entre el mercado de trabajo y el mercado de recursos humanos, es la que da origen a una baja o alta rotación de personal, es decir, el término de rotación de recursos humanos es la fluctuación de personal entre una organización y su ambiente.

En suma, para el modelo de la organización como un sistema, la rotación de personal no es una causa, sino un efecto, la consecuencia de ciertos fenómenos localizados interna o externamente en la organización sobre la actitud y el comportamiento del personal. Es, por tanto, una variable dependiente de aquellos fenómenos internos y externos de la organización. 
Dentro de los fenómenos internos que ocurren en la organización se citan los siguientes: la política salarial de la organización; la política de beneficios de la organización; las oportunidades de crecimiento profesional localizadas dentro de la organización; el tipo de relaciones humanas desarrolladas dentro de la organización; la cultura organizacional desarrollada dentro de la organización; la política de reclutamiento y selección de recursos humanos; y los criterios y programas de entrenamiento a los recursos humanos.

Para atender los problemas internos es importante efectuar entrevistas de salida, ya que la información obtenida facilita a la organización tener un diagnóstico adecuado de las fallas que presentó la organización, que tuvieron un impacto en la decisión del trabajador de abandonarla y tomar las medidas necesarias para su corrección. La utilización de entrevistas de salida se plantea junto con los procesos de inducción, una vez seleccionado el nuevo personal. De lo contrario, daría como resultado el establecimiento de una política inadecuada de recursos humanos que provocaría una actitud negativa del nuevo personal predisponiendo su retiro de la organización.

La aplicación del análisis de sistemas que permite poner en práctica una efectiva y constante evaluación del funcionamiento de la política de recursos humanos desarrollada por la organización (Davis K. Y Werther W, 2004) en cuanto a los procedimientos de: reclutamiento y selección; integración de personal recién admitido; remuneración; beneficios sociales; entrenamiento; movimiento planificado del personal; higiene y seguridad de trabajo; mantenimiento de disciplina y organización; relaciones formales e informales con los empleados; y evaluación del desempeño.

El empleo de una perspectiva de sistemas es muy útil para el manejo de la rotación de personal (Sánchez A. 2007). Pero no es suficiente cuando la gerencia o la administración de recursos humanos no puede esperar a contar con la retroalimentación para tomar decisiones sobre el problema de rotación de personal, por medio de las entrevistas de salida y de los mecanismos internos de evaluación de la organización y reaccionar sólo cuando el problema ha ocurrido y, en ese momento, actuar para su solución puede ser inapropiado y costoso.

Después de éste análisis teórico, observamos que todas las teorías tienen elementos reales de posibles causas de la rotación de personal en el sector textil, y aún cuando los dos últimos Modelos están más cerca de lo diagnosticado.

\section{La rotación y la insatisfacción laboral.}

Las investigaciones realizadas según Cuesta Armando, (2000) coinciden con los estudiosos de este tema, en demostrar que la rotación del personal está influenciada por la desmotivación y la insatisfacción laboral por lo que podemos inferir que los motivos que implican la salida de los trabajadores son fundamentalmente laborales y en la mayoría de los casos pueden ser detectados mediante el estudio de la fluctuación 
potencial por lo que si se toman las medidas necesarias se puede reducir la rotación real.

Tomando en cuenta que la rotación de personal tiene varios orígenes, de acuerdo con la Asociación Mexicana en Dirección de Recursos Humano (2004), algunas causas importantes en la inestabilidad laboral incluyen la falta de liderazgo de los jefes inmediatos, la inexistencia de canales adecuados de comunicación, los factores culturales así como el entorno del empleado.

Los modelos teóricos descritos resaltan la importancia de entender el problema de la rotación de personal partiendo de sus causas para comprender las consecuencias y con ello poder controlar dicho fenómeno. Por tanto este trabajo de investigación se nutre de estas teorías y confirma que la satisfacción laboral y el compromiso del personal son variables que afectan en forma directa en el tema que estamos analizando. Este problema también se asocia a la desproporción existente entre la carga de trabajo y los salarios, en consecuencia se trata de buscar una solución fuera del centro laboral. La insatisfacción en el trabajo, según Gerardo Recio (2008), se considera un antecedente del compromiso organizacional ligado con el ausentismo, el bajo desempeño, rotación del personal, entre otras variables relacionadas con el trabajo de las personas. Junto con el involucramiento en el trabajo, el desempeño, los estímulos y recompensas, identificación e internalización y el tipo de estímulos que pueden ser económica y psicológica en las que se han determinado correlaciones significativas con el compromiso hacia la organización.

Las políticas de personal, en las empresas son la solución, según los expertos, a la desmotivación que causa la rotación. Ya sean planes flexibles o bonos que mejoren el salario, programas de retención permiten a las compañías ahorrar gastos de capacitación de nuevos empleados por rotación.

Las empresas que trabajan en esta línea (políticas de administración de personal) no sólo reducen el nivel de rotación, sino que aumentan el de motivación, la productividad y bajan sus costos de capacitación y reclutamiento. Algunas empresas que se dedican a la subcontratación de personal para otras empresas, dicen que las principales razones por las que los trabajadores piensan en cambiar de rumbo antes de cumplir un año en la empresa para la que trabajan son: que el empleado haya tomado el empleo mientras encontrara algo mejor simplemente por urgencia de un ingreso o porque "buscar trabajo con trabajo es más fácil", que no se identifique con la cultura de la organización y/o con el liderazgo, que alguna promesa no se haya cumplido y se sienta defraudado.

Hay que proveer a los empleados de mejores oportunidades, desarrollo profesional, salarios competitivos o beneficios y un adecuado clima laboral, explican expertos directivos de empresas en de las entrevistas focalizadas que se llevaron a cabo. El $45 \%$ del personal permanecerá menos de seis meses en su empleo, informó Bumeran, la firma de recursos humanos y bolsa de trabajo en línea, en una encuesta. Mientras que $16.15 \%$ mantendrán su trabajo entre seis meses y un año y sólo $11.80 \%$ estima quedarse más de cinco años trabajando donde lo hace ahora. 
La rotación del personal puede causar problemas que cuesten a las empresas o a las oportunidades de los trabajadores. Una persona que está buscando un empleo mientras trabaja no es tan productiva como puede ser, lo que se traduce en pérdidas económicas para la empresa.

El índice de rotación de personal de posiciones profesionales en México tiene un promedio nacional de $5.76 \%$, según datos encontrados en agencias de colocación de empleos. Sin embargo, es mayor en la zona sureste del país (6.58\%) y menor en la región del valle (4.96\%). Por otro lado en España y en Europa, en general, tenemos una cultura que valora extraordinariamente la estabilidad en el empleo.

Muchos Directores de Recursos Humanos se muestran orgullosos al comentar que la rotación externa de su personal es cero o insignificante; eso es señal, dicen, de que el entorno, las condiciones de trabajo y la motivación de sus trabajadores es la adecuada y no tienen necesidad de buscar trabajo en otras empresas, aunque como lo constatamos anteriormente estas afirmaciones de los directores de recursos humanos no son necesariamente correctas, sobre todo en los tiempos actuales de crisis nacional y mundial, donde el desempleo y la falta de oportunidades esta también golpeando a los países del primer mundo.

\subsection{Modelo MORALE. Por Ahr y Ahr, 2000}

Ahr y Ahr proponen en su modelo MORALE que la rotación está relacionada de manera directa con las variables que se estudian en el modelo propuesto:

$\begin{array}{ll}\mathrm{E} & \text { Relaciones } \\ \mathrm{Q} & \text { Remuneración } \\ \mathrm{U} & \text { Oportunidades } \\ \mathrm{I} & \text { Adaptabilidad } \\ \mathrm{D} & \text { Liderazgo } \\ \mathrm{A} & \\ \mathrm{D} & \end{array}$

Fuente: Ahr, Ahr (2000)

Llaman a este modelo MORALE por que las variables que relacionan forman las siglas en inglés de esta palabra. Según estos autores, de estas variables dependerá la satisfacción, productividad y compromiso hacia la organización que el empleado cree. En resumen, afirman que las razones por las que un empleado deja la organización pueden ser sorteadas en las seis categorías. La adecuada remuneración deberá estar en la línea más baja del modelo porque para la mayor parte de empleados éste no es el factor más importante para decidir si permanecen o dejan la organización. 
Se toma del modelo MORALE las variables de percepción que se adaptan al modelo desarrollado en esta investigación.

En cambio, la percepción que ellos tengan de la equidad en el trabajo es una determinante de suma importancia en su decisión. Su preponderancia está basada en el hecho de que está mide el desempeño de todas las otras categorías.

5.2 Por Rodrigo Arrioja, 1993: Modelo simplificado multivariado para analizar la rotación en la industria maquiladora.

Rodrigo Arrioja incluye cuatro medidas de efectividad del área de recursos humanos y seis variables independientes. El diseño recae en un punto entre los modelo tempranos de recursos humanos bivariables y otros más sofisticados usados en ambientes altamente desarrollados en lo industrial.

Variables dependientes:

Rotación de personal: se toma como la tasa de rotación mensual de las empresas estudiadas.

Ausentismo: se toma como la tasa de ausentismo de las empresas estudiadas.

Accidentes; se toma como la tasa de accidentes de las empresas.

Quejas: se toma como la tasa de quejas de los empleados de las empresas estudiadas.

Variables independientes:

Género

Ambiente laboral

Actividad industrial

Origen del trabajador

Edad de la planta

Tamaño de la planta

Mercado laboral

Sindicalismo

5.3 Modelo de Rotación para la industria maquiladora de Chihuahua, México. Peña, 1999

Variable dependiente:

Tasa de rotación mensual de las empresas estudiadas

Variables independientes: 
Estado civil

Estado migratorio

Edad

Género

Educación

Experiencia de trabajo previa

Turno de trabajo

Actividad industrial de la empresa

Presencia y número de hijos

Contenido de trabajo

Cultura organizacional

Cultura nacional

Distancia del poder

Percepción del empleado sobre la remuneración que recibe.

En su modelo, Peña analiza la congruencia entre la cultura organizacional y nacional. Ella afirma que el perfil organizacional y las características de la empresa definen las causas de rotación, así como el contenido de trabajo y la percepción que el empleado tiene sobre la remuneración que recibe.

\section{Conclusión}

La industria del vestido en México se caracteriza por ser intensiva en mano de obra y por ser altamente heterogénea en cuanto a tamaño, calidad, marcado y organización. Actualmente es una de las más dinámicas y globalizadas del mundo. Sin embargo, hay empresas líderes -ubicadas principalmente en países desarrolladosdonde controlan el diseño, la comercialización y la marca.

A lo largo de este artículo se han revisado las distintas modalidades, conceptos y metodologías aplicadas para comprender el fenómeno de la rotación de personal en el contexto de la industria textil de Tlaxcala. Resulta evidente que los cambios constantes de trabajadores responde a múltiples factores: culturales, económicos y emotivos. En especial destaca el papel que desempeñan las estrategias de motivación instrumentadas para disminuir los índices de rotación de personal. Es por eso que las organizaciones empresariales deben comprender que más allá del compromiso contractual de pagar un salario por la labor cumplida, los trabajadores requieren un 
plan de carrera integral que mantenga las condiciones emocionales idóneas para trabajar con una actitud positiva e innovadora.

En la medida en que las empresas sean capaces de incorporar planes de carrera que establezcan procesos de capacitación, opciones de desarrollo humano integral y la integración de equipos de trabajo de alto rendimiento sujetos a procesos de reconocimiento de los logros que sean transparentes y justos, en esa misma medida aumentará la percepción de bienestar de los trabajadores, su motivación y así garantizar una mayor estabilidad de sus colaboradores para disminuir el impacto de la rotación de personal en la productividad de la industria textil tlaxcalteca.

\section{Bibliografía}

ALVARADO R. C. Y VIEYRA, A. (2002) "La Subcontratación de las Grandes Empresas de la Confección en la Zona Metropolitana de la Ciudad de México", Problemas de Desarrollo, vol. 33, no. 130.

ARRIOJA R. (1993) The North American Free Trade Agreement and its implications for human resources management. Colorado, pp. 35-37

AZTEX TRADING Y SECRETARÍA DE ECONOMÍA (2006) Estudio de Identificación de Vocación Productiva Actual de la Industria de la Confección, para Adecuar la Oferta de Teletecas, México.

CARRILLO, J. Y SANTIBÁÑEZ, J. (2001). Rotación de Personal en las maquiladoras $\left(2^{\mathrm{a}}\right.$ ed.) México: el Colegio de la Frontera Norte, pp. 13

CASCIO, W. F (1991) Costing Human Resources ( $3^{\mathrm{a}}$ ed.) New York: MCGraw-Hill, p. 52

CHIAVENATO I. (2009) Comportamiento Organizacional. La dinámica del éxito en las organizaciones. México: McGraw Hill

CONOCER, (2000) Análisis Sectorial de la Industria Textil y del Vestido, México: Limusa.

DAVIS K. Y WERTHER, W. B.(2000) Administración de Personal y Recursos Humanos, México: McGraw-Hill.

DAVISON P. L. G. (2002) La Actividad Textil de un Pueblo Nahua de La Malinche, México: Cuso.

DELAUNAY, M. (2005) "El Hilo Negro que Todos Quieren", Expansión, vol. XXXVI, no. 921.

DUSSEL P. E. Y CÁRDENAS C. H. (2007) "México y China en la Cadena Hilo-Textil-Confección en el Mercado de Estados Unidos", Comercio Exterior, vol. 57, no. 7.

G. GEREFFI, (2000) "La Transformación de la Industria de la Indumentaria en América del Norte: ¿Es el TLC una Maldición o Castigo?”, en Impacto del TLC en las Exportaciones de Prendas de Vestir de los Países de América Central, República Dominicana y México, R. Buitelar y E. Rodríguez (ed.), Santiago de Chile: CEPAL. 
GEREFFI, G. (2001) "Las Cadenas Productivas como Marco Analítico para la Globalización", Problemas de Desarrollo, vol. 32, no. 125, México: IIEC-UNAM.

GEREFFI, G. (2006) The Fiber-Textile-Apparel Value Chain: Mexico and China Compared, First on Opportunities in the Economic and Trade Relationship between China and Mexico in a Latin America Context.

GÓMEZ-MEJÍA, L. BALKIN D. Y CARDY. (2008) Gestión de los Recursos Humanos. Madrid. Pearson Prentice Hall. pp. 228-230

HOLTOM C. B. MITCHELL R. T., LEE W T., EBERLY B. M. (2008) Turnorver and Retention Research: A Glance at Pst, a Closer Review of the Present, and a Venture into the Future. The Academy of Management Annals. Vol 2, No, 1, pp. 231-274.

KACMAR, M. BOZEMAN, D. DAWN; WILLIAM A. (1999). An Examination of the PErceptions of Organizational Politics Models: Replicaton and Extension. Plenum Publishing Corporation.

PEÑA, L (2000). Retaining a MExican Labor Force, Journal of Business Ethics, pp. 123-131

RODRÍGUEZ, PINTO Y NÚÑEZ, (2012), "Estrategia de gestión de aprendizaje en la red de actores del sector agropecuario en el municipio Mayarí en cuba", en revista SEECI, noviembre, Madrid.

ROBBINSON P. S. Y JUDGE A. T. (2009) Comportamiento Organizacional. México: Pearson Prentice Hall.

RUEDA P. I. Y DOMÍNGUEZ N. S.(comp.), (2006) El Dilema de la Industria del Vestido en México: Los Casos de Aguascalientes y Yucatán, México: UNAM Porrúa.

SÁNCHEZ S. A. (2007) Globalización e Industria de la Confección: Análisis de las Condiciones que Globalizan la Industria de la Confección Mexicana a Partir del Caso Tlaxcalteca, México: UAT.

TAMAYO C.P. (2008) Modelos Teóricos e Investigación sobre Rotación de Personal, México: PAC.

VIGODA-GADOT E. (2006) Citizen's perceptions of Politics and Ethics in Public Administration: A Five-Year National Study of Their Relationship to Satisfaction with Services, Trust in Governance, and orientations. Oxford University Press 